\title{
Rheological properties, oxidative stability and sensory evaluation of enzymatically synthesized medium- and long-chain triacylglycerol-based salad dressings
}

\begin{abstract}
The rheological properties of various oil-based salad dressings were investigated. The shear rate-shear stress study based on Power Law's model indicated pseudoplastic fluid behaviors of dressings prepared in this study. Soybean- and medium- and long-chain triacylglycerols (MLCT)-based dressings treated with different antioxidants showed similar rheological behaviors based on the changes in the storage modulus, loss modulus, viscosity profile and loss tangent in the frequency, amplitude and temperature sweep study. The time-dependent study revealed similar thixotrophic properties between soybean- and MLCT-based dressings. Addition of synthetic or natural antioxidants reduced the rate of oil deterioration in oil-inwater emulsion as a function of time. No significant differences $(p>0.05)$ in hydrolytic reaction of all dressing systems were observed throughout the 3 months of storage at $20{ }^{\circ} \mathrm{C}$. Quantitative descriptive analysis using trained panelists revealed no significant differences ( $p>0.05$ ) either in salad oils or salad dressings except for MLCT-based oils/dressings treated with oleoresin sage extracts, which showed darker and stronger odor intensity than other oil systems. Nevertheless, the overall quality of the physical appearance and organoleptic acceptability showed no significant differences $(p>0.05)$ in all systems, indicating that MLCT-based blends can be used as healthy functional oils to replace soybean oil in food dressing applications.
\end{abstract}

Keyword: Medium and long chain triacylglycerols; Oleoresin sage extracts; Rheological properties; Tert-butylhydroquinone; Salad dressings 